\section{ROYAL SOCIETY OF CANADA MEETING AT LAVAL UNIVERSITY,
QUEBEC}

$\mathrm{T}$ HE Royal Society of Canada held its annual meeting at Laval University, Quebec, during June 2-4. In Section III (Chemical, Mathematical and Physical Sciences) of the Society, the following new Fellows were elected: P. E. Gishler, L. Katz, W. B. Lewis, D. W. R. McKinley, P. Scherk and H. L. Welsh. Dr. G. Herzberg gave his presidential address on "Forbidden Transitions in Diatomic Molecules". This was one of three interesting papers in a symposium on forbidden transitions. The others were "Forbidden Transitions in Atoms", by A. G. Shenstone, and "Forbidden Transitions in Nuclei", by L. G. Elliott.

About a hundred papers were presented before the sub-sections-chemistry, mathematics, astrophysics and spectroscopy, nuclear physics, meteorology and other branches of physics.

Chemistry. The programme of the chemistry subsection consisted of a series of papers in organic chemistry (P. E. Gagnon et al.) and several in physical chemistry. The photolysis of azomethane has been investigated over the temperature-range $25^{\circ}-190^{\circ} \mathrm{C}$. (M. H. Jones and E. W. R. Steacie). Experiments on the photolysis of deuterated methylamines have shown that both the $\mathrm{C}-\mathrm{H}$ and $\mathrm{N}-\mathrm{H}$ bonds are ruptured (J. S. Watson and B. deB. Darwent). Various forms of deuterated pentanone- 3 have been synthesized (L. C. Leitch and A. T. Morse). The assignments of certain infra-red absorption bands between 1,350 and $1,475 \mathrm{~cm}^{-1}$ to the bending vibrations of the $\mathrm{C}-\mathrm{H}$ bonds of methyl and methylene groups located at specific positions in the molecule have been confirmed in forms of deuterated pentanone-3 (B. Nolin).

Mathematics. Three papers were presented in algebra and number theory, eight in analysis, and two in geometry. The significance of the work presented and the general interest and enthusiasm made this meeting the most outstanding of any yet held by the mathematics sub-section. It was generally agreed that the upsurge of activity on the part of Canadian mathematicians is due to the stimulus of the biennial seminars of the Canadian Mathematical Congress and especially to the influence of the Summer Research Institute.

Astrophysics and Spectroscopy. The astronomical papers included a discussion of the determination of the masses of eclipsing binaries, the study of interstellar matter, the investigation of the line profiles of Be-type stars, and the development of new techniques for the determination of time. The orbital elements of the exceptionally massive eclipsing system, H.D. 228854, have been deduced (J. A. Pearce). These stars are of special class $O 7$ with temperatures of $33,000^{\circ} \mathrm{K}$. The orbital inclination is nearly $90^{\circ}$, and the relative orbital velocities are exceptionally large $(710 \mathrm{~km}$. $/$ sec.). The combined mass is seventy times the sun. A new catalogue of 1,346 variable stars in globular clusters has been prepared (Helen S. Hogg). Investigation of the absorption lines of the eclipsing stars Zeta Aurigæ when light of the hot star traverses the atmosphere of the larger and cooler companion has provided evidence of major prominence activity of irregular character (A. McKeller and R. M. Petrie). The intensities of interstellar sodium and calcium lines for 142 early-type stars have been used to derive a relation between distance and intensity (C. S. Beals).

Relatively high-dispersion spectra of the aurora have been obtained in the region 3300-8900 A. (W. Petrie). The near-ultra-violet emission bands of the $\mathrm{N}_{2}+$ molecule support the value $9 \cdot 75 \mathrm{eV}$. for the dissociation energy (A. E. Douglas). The rates of change of polarizability with respect to internuclear distance have been found from the ratio of the intensities of Raman and Rayleigh scattering in gases (E. J. Stansbury, H. L. Welsh and M. F. Crawford). Experimental and theoretical results on the splitting of nuclear magnetic resonance absorption lines in single crystals by the interaction of the nuclear electric quadrupole moment with the electric field gradient within the crystal were reported (G. M. Volkoff).

Nuclear Physics. A heavy and varied programme of nuclear physics papers on heavy cosmic primaries in photographic emulsions, photonuclear reactions, neutron physics, radioactive nuclides, and mass spectrometry was presented.

Several papers on neutron physics from the Chalk River Laboratory will be briefly reviewed. Work on the resonant scattering of slow neutrons by samarium, gadolinium, europium, dysprosium and cadmium was reported. The scattering and total cross-sections of cadmium in the resonance region accurately obey the Breit-Wigner one-level formulæ. The total crosssection at resonance is 7,900 barns (B. N. Brockhouse). The neutron capture $\gamma$-ray spectra from the evencharge nuclei titanium, chromium, iron, nickel and zinc, and from the odd-charge nuclei scandium, manganese, cobalt and copper, were shown and discussed in terms of nuclear shell theory (B. B. Kinsey and G. A. Bartholomew). The angular distributions of prompt fission neutrons with respect to the direction of motion of the light and heavy fragments from the thermal neutron fission of uranium$233,-235$ and plutonium-239 show that the neutron emission probability is 30 per cent greater for the light fragment (J. S. Fraser and J. C. D. Milton). The neutron capture cross-section for ${ }^{59} \mathrm{Co}(n, \gamma)$ ${ }^{60 \mathrm{~m}} \mathrm{Co}(10.7 \mathrm{~min}$.) was reported as 19 barns (N. Moss and $\mathrm{L}$. Yaffe). The nuclide radium-227, produced by the ${ }^{22} \operatorname{Ra}(n, \gamma)^{222} \mathrm{Ra}$ reaction with a cross-section of 22 barns, is a $\beta$-emitter with a half-life of $41.2 \mathrm{~min}$., a maximum energy of $1.30 \mathrm{MeV}$., and $\gamma$-rays of approximately 291 and $497 \mathrm{keV}$. (J. P. Butler and J. S. Adam). A curious time behaviour of positons annihilated in liquids and solids was reported. In metals and several other materials, positons are annihilated with a single mean life-time of about $2 \times 10^{-10} \mathrm{sec}$. In other materials (for example, water, ice) two distinct life-times were observed-about $3 \times 10^{-10}$ sec. and $2 \times 10^{-9}$ sec. (R. E. Bell and R. L. Graham).

Papers on the radioactive properties of neutrondeficient nuclides, cadmium-105 and -104 , silver-104, xenon-123, -122 and -121 , produced by proton bombardment in the McGill University cyclotron, were read (J. S. Foster, F. A. Johnson and D. E. Tilley). The X-rays from the 70-MeV. synchrotron at Queen's University have been used to study depth-dose relations in a 'Plexiglas' phantom, the absorption of $17 \cdot 5-\mathrm{MeV}, \mathrm{X}$-rays in various absorbers with the aid of ${ }^{63} \mathrm{Cu}(\gamma, n)^{62} \mathrm{Cu}$ as detector, and the production and 
cross-sections of photonuclear reactions such as the $(\gamma, n)$ reactions in carbon-12, copper-63, silver-107 and molybdenum-92 and the reactions ${ }^{12} \mathrm{C}(\gamma, 3 \alpha)$ and ${ }^{16} \mathrm{O}(\gamma, 4 \alpha)(J$. A. Gray et al. $)$. The $25-\mathrm{MeV}$. betatron at the University of Saskatchewan can be held constant to $5 \mathrm{keV}$. at a pre-selected energy. Photoactivation curves have been obtained which show a fine-structure that is attributed to absorption by individual levels in the reacting nucleus. The ratios of the cross-sections for the $(\gamma, n)$ reactions leading to the ground and isomeric states in bromine- 80 and molybdenum-91 have been measured and related to the spins of the reacting particles (L. Katz, R. N. H. Haslam et al.). Excellent agreement with experimental results on the capture of electrons by protons in hydrogen is obtained if both the ion - nucleus interaction and the electron - ion interaction are included as perturbations in a calculation using the Born approximation (J. D. Jackson and H. Schiff).

Other Branches of Physics. Papers in meteorology included a discussion of the conditions for the production of low-temperature fog (W. L. Godson), a correlation between types of snow crystal and the temperature at the height of formation (L. W. Gold and B. A. Power), and evaluation of fluctuating radar weather echoes (J. S. Marshall and W. Hitschfeld). A method for measuring sub-surface velocities in the Gulf Stream was described (E. E. Watson). A flowmeter, utilizing the cooling of a thermistor element, has been found suitable for measurement of small velocities in liquids (A. D. Misener).

A number of papers were presented on the properties of dielectrics, theory of diffraction, sources of sound, vibration of plates, infra-red detection, and an absolute measurement of the ohm. The failure of the Clausius-Mosotti equation for certain artificial dielectrics was explained in terms of the random distribution and shape of the metallic particles (H. E. J. Neugebauer). Discrepancies between diffraction theories and measurements close to a circular aperture under the influence of a plane acoustic wave have been traced to rapid oscillations in field intensity within the aperture (G. Bekefi). An improved method of calculating the diffraction of electro-magnetic waves by small apertures and scattering by small conducting bodies was reported (A. F. Stevenson). Calculations and observations have been made on the vibration patterns of thick barium titanate disks (R. R. Aggarwal and E. A. G. Shaw). The usefulness of physical theory in solving engineering problems was well demonstrated in a study of chopper-type sources of sound for fog-horns and air-raid sirens (G. J. Thiessen).

Three papers on low-temperature and solid-state physics were read (D. K. C. MacDonald). By suitably modifying a Collins helium liquefier, continuous measurements of electrical resistances of metals over a wide range of temperature have been made. The variation of characteristic temperature has been examined and carefully compared with the corresponding calorimetric data. The problems of anharmonicity in lattice vibrations and of melting are of particular interest, together with the adequacy or otherwise of the Debye model. The anomalous minimum of electrical resistance found at about $10^{\circ} \mathrm{K}$. in copper has been systematically investigated with specimens containing controlled small percentages of tin, silver, nickel, lead, bismuth and carbon. Scattering cross-sections can be deduced from the residual resistances. B. W. SARGENT

\section{EMPLOYMENT PROBLEMS OF DISABLED YOUTH IN GLASGOW}

TNCREASING concern about the employment of the disabled led, in 1944, to the passing of the Disabled Persons (Employment) Act. It was provided in the Act that the Ministry of Labour should establish and maintain a voluntary register of disabled people and that the larger firms should be obliged to employ a quota of workers who were registered in this way. The Act also gave the Ministry power to set up units to train or rehabilitate the disabled.

The practical application of these measures brought many difficulties to light and made it clear that much more detailed information was needed about the medical problems of placing disabled people in employment. In 1948, therefore, the Ministry of Labour asked the Medical Research Council to help. The Council's Occupational Medicine Committee agreed that the first necessity was to collect information about the incidence of disability and the extent to which the various forms of disability interfered with employment, and on its recommendation the Council set up a special Committee on the Resettlement of the Disabled.

A memorandum has now been prepared recording an investigation which was carried out under the ægis of this Committee into the employment experience of two groups of young people in Glasgow ; one, a group of registered disabled, the other made up of children who had been to special schools for the physically handicapped*.

In the first group, 683 young persons were registered as disabled and, of these, 104 were not covered by the investigation for various reasons. Of the remaining 579, 146 had infective and parasitic diseases and 164 haid diseases of the nervous system. About 18 per cent of the group were found to be unemployed and, of those in work, the majority were employed as semi-skilled manual workers, although quite a number were employed as skilled workers in crafts like boot repairing, cabinet making, market gardening, painting, watch repairing, upholstering, glazing, sheet metal work, baking and bookbinding. Unemployment was noticeably high among the group disabled by diseases of the nervous system; this group included epileptics and congenital paraplegics. T'he groups disabled by tuberculosis and mental defect showed degrees of unemployment that were substantially above the average. The groups disabled by malformations, poliomyelitis, diseases of the ear and of bones all showed low unemployment-rates. Many of the young people disabled by congenital malformations, accidents, circulatory diseases and poliomyelitis were engaged in non-manual work, while a relatively high proportion of those disabled by poliomyelitis and deafness secured skilled manual work.

The general employment picture was most favourable among those disabled by poliomyelitis and deafness. In the former group this is probably because the disability is not progressive, seldom affects more than one member of the family, and does not often involve the loss of many years schooling. There is a belief that the character of the poliomyelitic patient tends to be robust and free from self-pity. In this survey the way in which they regarded their disability was outstandingly healthy

* Fmployment Problems of Disabled Youth in Glasgow. (London : J.M.S.O.) $3 s$. 